\title{
Relating and contrasting plain and prefix Kolmogorov complexity
}

\author{
Bruno Bauwens*
}

We present a short proof of Solovay's results from [5] relating plain and prefix complexity:

$$
\begin{aligned}
& K(x)=C(x)+C C(x)+O(C C C(x)) \\
& C(x)=K(x)-K K(x)+O(K K K(x)),
\end{aligned}
$$

(here $C C(x)$ denotes $C(C(x))$, etc.).

In [3] a short proof is given that some strings have maximal plain Kolmogorov complexity but not maximal prefix-free complexity. The same proof technique was applied to solve three open problems (see [2]):

1) There exist a sequence $\omega$ such that $\liminf C\left(\omega_{1} \ldots \omega_{n}\right)-C(n)$ is infinite and $\lim \inf K\left(\omega_{1} \ldots \omega_{n}\right)-K(n)$ is finite, i.e. the infinitely often $C$-trivial reals are not the same as the infinitely often $K$-trivial reals, (i.e. [1, Question 1]).

2) The 2-random sequences are exactly those sequences that have infinitely many initial segments with maximal plain complexity. This also holds for prefix complexity. We show that some initial segments of a 2-random sequence with maximal plain do not have maximal prefix complexity (a question from $\mathrm{L}$. Bienvenu).

3) We show that there exist no monotone relation between probability and expectation bounded randomness deficiency, (i.e. [4, Question 1]). Because of its simplicity, we present this proof.

If time permits, we discuss an unrelated negative result. Van Lambalgen theorem is shown for Martin-Löf randomness relative to computable measures: a pair of sequences $(\alpha, \beta)$ is random if and only if $\alpha$ is random and $\beta$ is random relative to $\alpha$. We argue that the theorem can not be generalized for randomness relative to a computable measure (a question from A. Shen and H. Takahashi [6]).

\section{References}

[1] G. Barmpalias. Algorithmic randomness and measures of complexity. The Bulletin of Symbolic Logic, 19(3), Sept. 2013.

[2] B. Bauwens. Relating and contrasting plain and prefix Kolmogorov complexity. ArXiv e-prints, November 2013.

\footnotetext{
*Independent researcher
} 
[3] B. Bauwens and A. Shen. Complexity of complexity and maximal plain versus prefix-free Kolmogorov complexity. Journal of Symbolic Logic, 2013. Accepted for publication.

[4] L. Bienvenu, P. Gács, M. Hoyrup, C. Rojas, and A. Shen. Algorithmic tests and randomness with respect to a class of measures. Proceedings of the Steklov Institute of Mathematics, 274(1):34-89, 2011.

[5] R.M. Solovay. Draft of a paper (or series of papers) on Chaitin's work. 215 pp., unpublished, May 1975.

[6] Hayato Takahashi. Generalization of van lambalgen's theorem and blind randomness for conditional probability. arXiv preprint arXiv:1310.0709, 2013. Presented in sept 2013 at CCR in Moscow. 